\title{
Pharmacodynamics of Rocuronium in Cholestatic Patients with or without Hepatocellular Injury: Normal Onset Time of Initial Dose and Prolonged Duration Time After Repeated Doses
}

\author{
Jian-jun Yang ${ }^{1}$, Yong-guang Wang ${ }^{1}$, Zhuan Zhang ${ }^{1}$, Zhi-jie Zhang ${ }^{1}$, Jin Liu ${ }^{2}$, Jian-guo Xu ${ }^{1}$ \\ ${ }^{1}$ Department of Anesthesiology, Jinling Hospital, School of Medicine, Nanjing University, Nanjing, P.R. China \\ ${ }^{2}$ Department of Anesthesiology, West China Hospital, Sichuan University, Chengdu, P.R. China
}

Received, June 2, 2007, Revised, August 30, 2007; Accepted, June 13, 2008, Published June 19, 2008.

\begin{abstract}
Purpose. A prospective controlled study was designed to observe the pharmacodynamics of rocuronium in cholestatic patients with or without hepatocellular injury.
\end{abstract} Methods. Sixty patients undergoing abdominal surgery were allocated into three groups: group I had 20 cholestatic patients with hepatocellular injury; group II had 20 cholestatic patients without hepatocellular injury, and group III (control group) had 20 patients without hepatic disease. Anesthetized with propofol and fentanyl, all patients received rocuronium $0.6 \mathrm{mg} / \mathrm{kg}$ for initial dose followed by intermittent repeated administration of rocuronium $0.15 \mathrm{mg} / \mathrm{kg}$. The twitch high of adductor pollicis muscle was monitored by acceleromyography. The onset time of the initial dose, the duration time of the initial and the repeated doses, and the recovery index were observed. Results. The onset and the duration time of the initial dose had no significant difference among the three groups $(\mathrm{P}>0.05)$. After administration of the $5^{\text {th }}$ dose, the duration time of the repeated doses was significantly prolonged than that of the $2^{\text {nd }}$ dose in group I ( $31 \pm 8$ versus $22 \pm 4 \mathrm{~min})$ and group II $(28 \pm 5$ versus $21 \pm 4 \mathrm{~min})(\mathrm{P}<0.05)$, but not in group III $(\mathrm{P}>0.05)$. The recovery index of rocuronium was longer in group I (48 $\pm 13 \mathrm{~min})$ and group II (46 \pm 9 $\mathrm{min})$ than that in group III $(24 \pm 5 \mathrm{~min})(\mathrm{P}<0.05)$. Conclusion. Cholestatic patients experience prolonged duration time and longer recovery index after repeated use of rocuronium, despite normal onset time after the initial dose.

\section{INTRODUCTION}

Rocuronium, an intermediate acting nondepolarizing steroidal neuromuscular blocking agent (NMBA) with a rapid onset time, can provide good intubating conditions within 60 to $90 \mathrm{~s}$ after rocuronium $0.6 \mathrm{mg} / \mathrm{kg}$ (1-3). Rocuronium is eliminated by the liver (3-6) and the kidney (3-5), and the former plays a more significantly important role (1-6).

Patients with choledocholithiasis often need surgical treatment under general anesthesia, in which rocuronium is usually used (7). Cholestasis, as a result of obstructive bile duct induced by choledocholithiasis, is a common disease with modest liver dysfunction (7-8). Rocuronium has been studied extensively in patients with severe liver dysfunction particularly cirrhosis (9-11). However, the pharmacodynamics of rocuronium in patients with choledocholithiasis inducing cholestasis remains unknown. Since rocuronium is mainly excreted by the liver (3-5), we hypothesized that the pharmacodynamics

Corresponding Author: Jin Liu, MD, Department of Anesthesiology, West China Hospital, Sichuan University, Chengdu 610041, P.R. China. E-mail: yjyangjj@126.com 
of rocuronium is changed in choledocholithiasis inducing cholestatic patients, and designed a prospective controlled study to examine the characteristics of the pharmacodynamics of rocuronium in choledocholithiasis induced inducing cholestatic patients with or without hepatocellular injury.

\section{METHODS}

\section{Ethics Consideration}

The present study was approved by the Ethics Committee of Jinling Hospital and conducted according to the Helsinki Declaration, and prior written informed consents were obtained from all the patients.

\section{Inclusion and Exclusion Criteria of Patients}

Sixty adult patients, 20 to 50 years of age, undergoing scheduled elective abdominal surgeries (choledocholithotomy, Roux-en-Y gastric bypass surgery, or gastrectomy) were enrolled in the present study. They were divided into three groups: group I and group II had 20 cholestatic patients with or without hepatocellular injury [with or without a higher glutamate-pyruvate transaminase (GPT) and glutamine oxaloacetic transaminase (GOT)], respectively; and group III, served as control group had 20 age, sex, and body mass index (BMI) matched patients without hepatic disease. The patients in group I and group II, suffering from choledocholithiasis associated with cholangiectasis, underwent choledocholithotomy or Roux-en-Y gastric bypass surgery, and the patients in group III underwent gastrectomy.

Patients with cirrhosis, hepatitis, hepatoma, drug-induced or other forms of cholestasis, heart failure or arrhythmia, renal dysfunction, water-electrolyte disturbances, acid-base imbalances, or neuromuscular disorders were excluded from the present study. None of the patients received any other drugs known to interact with neuromuscular blocking drugs before surgery.

Demographics and the laboratory test results of hepatic function were recorded when patients were preoperatively interviewed the day before surgery.

\section{Anesthesia Techniques}

All the patients were premedicated with intramuscular phenobarbital $0.1 \mathrm{~g}$ and scopolamine $0.3 \mathrm{mg}$ half an hour before anesthesia. After the patients were admitted to the operation room, electrocardiography, pulse oximetry saturation, and invasive blood pressure through left radial artery cannulation were monitored. After left cephalic vein cannulation, Ringer's lactate was intravenously infused at the rate of $10 \pm 2 \mathrm{ml} / \mathrm{kg} / \mathrm{h}$.

General anesthesia was induced with midazolam $0.05 \mathrm{mg} / \mathrm{kg}$, fentanyl $3.0 \mu \mathrm{g} / \mathrm{kg}$ and propofol 1.5-2.5 mg/kg. Rocuronium (Organon, Holland) $\quad 0.6 \quad \mathrm{mg} / \mathrm{kg}$ was intravenously administered within $3 \mathrm{~s}$ to facilitate endotracheal intubation, which was followed by intermittent administration of rocuronium $0.15 \mathrm{mg} / \mathrm{kg}$ for maintenance doses. After endotracheal intubation, all the patients were mechanically ventilated to keep the end tidal carbon dioxide tension 30-35 mmHg. Anesthesia was maintained with continuous intravenous infusion of fentanyl 1.0-2.0 $\mu \mathrm{g} / \mathrm{kg} / \mathrm{h}$ and propofol $5-10 \mathrm{mg} / \mathrm{kg} / \mathrm{h}$ adjusted on the basis of physiological signs, such as heart rate, arterial blood pressure, and pupil size.

\section{Neuromuscular Blocking Effects}

Neuromuscular transmission was monitored by acceleromyography (NMT mechanosensor) using train-of-four-sequence (TOF) (Datex-Ohmeda S/5 
Anesthesia Monitor, Datex-Ohmeda Divisin, Instrumentarium Corp., Helsinki, Finland). The right forearm was prepared for monitoring. Skin temperature was monitored and maintained above $32^{\circ} \mathrm{C}$ with the room air temperature controlled at about $24{ }^{\circ} \mathrm{C}$. The hand and the forearm were immobilized in a splint allowing free mobility of the thumb and the neuromuscular stabilization took place before administration of rocuronium. The monitor automatically determined the supramaximal current and response reference level, and then the ulnar nerve was stimulated at the wrist via surface electrodes by supramaximal current in a TOF $(0.2 \mathrm{~ms}$ duration, $2 \mathrm{~Hz}$, four consecutive square wave impulses). These stimuli were delivered continuously to determine the onset time of the initial dose and then every $20 \mathrm{~s}$ to determine the duration time and the recovery index. To determine the onset time and the recovery of rocuronium, the response of the adductor pollicis muscle to stimulation, which was quantified using an acceleromyographic probe fixed to the volar surface of the distal phalanx of the thumb, of the ulnar nerve was monitored. The TOF monitor was connected to a personal computer for online data recording and processing.

The time from initial administration of rocuronium to endotracheal extubation was monitored. The effects of NMB were monitored as the following steps: 1) recorded the time from the end of rocuronium initial $\left(1^{\text {st }}\right)$ administration to complete or the maximal depression of the first twitch (onset time); 2) recorded the time from the $1^{\text {st }}$ administration to the recovery of the first twitch of the TOF response to $\left.25 \%\left(1^{\text {st }} \mathrm{T} 25\right) ; 3\right)$ once the recovery of the first twitch of the TOF response achieved $25 \%$, maintenance doses of rocuronium $0.15 \mathrm{mg} / \mathrm{kg}$ were immediately administered; 4) recorded the time from rocuronium $2^{\text {nd }}, 3^{\text {rd }}, 4^{\text {th }}, 5^{\text {th }}$, and $6^{\text {th }}$ administration (every patient received $6^{\text {th }}$ administration whether the surgery was over before $6^{\text {th }}$ administration or not) to the recovery of the first twitch of the TOF response to $25 \%\left(2^{\text {nd }}, 3^{\text {rd }}, 4^{\text {th }}, 5^{\text {th }}\right.$, and $\left.6^{\text {th }} \mathrm{T} 25\right)$, respectively; 5) recorded the recovery index (the time from $6^{\text {th }} \mathrm{T} 25$ to $6^{\text {th }} \mathrm{T} 75$ ).

\section{Statistical Analysis}

Data were expressed as mean \pm SD except that sex distribution was expressed as proportion. Statistical analysis was performed by a computer program, Statistical Product for the Social Sciences (SPSS version 11.5). Differences were considered to be significant at $\mathrm{P}<0.05$.

Sex distribution was compared using Chi-square between groups. After a test for homogeneity of variances, inter-group comparisons were performed using one-way analysis of variance (ANOVA) followed by Student-Newman-Keuls tests for post-hoc multiple comparisons (equal variances) or using nonparametric Kruskal-Wallis tests followed by Conover's $t$ tests for multiple comparisons of observations with unequal variances. Intra-group comparisons were performed using one-way ANOVA followed by Student-Newman-Keuls tests for post-hoc multiple comparisons.

\section{RESULTS}

\section{Study Population}

Demographics, the preoperative laboratory test results of hepatic function, and the body temperature $(\mathrm{T})$ at the end of the trial were compared between groups (Table 1). Patients in the three groups did not differ with respect to age, sex, body mass index (BMI), and $\mathrm{T}$ at the end of the trial $(\mathrm{P}>0.05)$, but differed with respect to total bilirubin, GPT, GOT, $\gamma$-glutamyl transpeptidase $(\gamma-\mathrm{GT})$, and alkaline phosphatase (AKP) $(\mathrm{P}<0.05)$. Total bilirubin, GPT, GOT, $\gamma$-GT, and AKP (Normal range in our hospital were $<19 \mu \mathrm{mol} / \mathrm{L}, 2-50 \mathrm{U} / \mathrm{L}, 2-50 \mathrm{U} / \mathrm{L}, 2-50 \mathrm{U} / \mathrm{L}$, 
and 50-172 U/L, respectively.) were significantly higher in group I than group II and group III $(\mathrm{P}<0.05) . \quad \gamma$-GT and AKP were significantly higher in group II than group III $(\mathrm{P}<0.05)$

(Table 1).

\section{Onset and Duration time of Rocuronium}

The assessments of neuromuscular blockade and recovery of rocuronium were recorded and statistically analyzed (Table 2). There was no significant difference between group I and group II.

Patients in group I and group II did not differ from those in group III with respect to the onset time, $1^{\text {st }}, 2^{\text {nd }}, 3^{\text {rd }}$, and $4^{\text {th }} \mathrm{T} 25(\mathrm{P}>0.05)$, but differed with respect to the $5^{\text {th }}$ and $6^{\text {th }} \mathrm{T} 25$ and the recovery index $(\mathrm{P}<0.05)$. The $5^{\text {th }}$ and $6^{\text {th }} \mathrm{T} 25$ were significantly longer in group I ( $31 \pm 8$ and $38 \pm 11$ $\mathrm{min})$ and group II $(28 \pm 5$ and $33 \pm 9 \mathrm{~min})$ than those in group III $(21 \pm 5$ and $23 \pm 5 \mathrm{~min})$, respectively $(\mathrm{P}<0.05)$. The $5^{\text {th }}$ and $6^{\text {th }} \mathrm{T} 25$ were also significantly longer than the $2^{\text {nd }} \mathrm{T} 25$ in group I ( $31 \pm 8$ and $38 \pm 11 \mathrm{~min}$ versus $22 \pm 4 \mathrm{~min}$ ) and group II ( $28 \pm 5$ and $33 \pm 9 \mathrm{~min}$ versus $21 \pm 4 \mathrm{~min}$ ), respectively $(\mathrm{P}<0.05)$. The recovery index of rocuronium was significantly prolonged in group I (48 $\pm 13 \mathrm{~min})$ and group II (46 $\pm 9 \mathrm{~min})$ than that in group III $(24 \pm 5 \mathrm{~min})(\mathrm{P}<0.05)$. (Table 2$)$

\section{DISCUSSION}

The patients in group I and group II, suffering from choledocholithiasis associated with cholangiectasis, having a higher total bilirubin, $\gamma$-GT and AKP indicated these patients experienced cholestasis $(7,8)$. A higher total bilirubin and liver enzyme, particularly the higher GPT and GOT, in group I represented significant hepatocellular injury in the patients in group I $(7,8)$.

Table 1. Demographics, preoperative laboratory test results of hepatic function, and body temperature at the end of the trial in cholestatic patients with hepatocellular injury (I), cholestatic patients without hepatocellular injury (II), and control group (without hepatic disease) (III).

\begin{tabular}{lccc}
\hline & Group I $(\mathrm{n}=20)$ & Group II $(\mathrm{n}=20)$ & Group III (n=20) \\
\hline Age $(\mathrm{yr})$ & $39 \pm 10$ & $36 \pm 10$ & $38 \pm 8$ \\
Sex $(\mathrm{M} / \mathrm{F})$ & $13 / 7$ & $11 / 9$ & $12 / 8$ \\
BMI $\left(\mathrm{kg} / \mathrm{m}^{2}\right)$ & $22.6 \pm 1.9$ & $23.0 \pm 2.2$ & $22.8 \pm 1.9$ \\
Total bilirubin $(\mu \mathrm{mol} / \mathrm{L})$ & $226 \pm 69 \# *$ & $73 \pm 19$ & $<19$ \\
GPT $(\mathrm{U} / \mathrm{L})$ & $159 \pm 52 \# *$ & $39 \pm 7$ & $31 \pm 7$ \\
GOT $(\mathrm{U} / \mathrm{L})$ & $106 \pm 31 \# *$ & $38 \pm 8$ & $29 \pm 10$ \\
$\gamma-\mathrm{GT}(\mathrm{U} / \mathrm{L})$ & $578 \pm 125 \# *$ & $226 \pm 72 \#$ & $27 \pm 12$ \\
AKP $(\mathrm{U} / \mathrm{L})$ & $358 \pm 87 \# *$ & $207 \pm 43 \#$ & $67 \pm 19$ \\
T at the end of the trial $(\quad)$ & $36.4 \pm 0.3$ & $36.2 \pm 0.3$ & $36.3 \pm 0.3$ \\
\hline
\end{tabular}

Data were expressed as mean \pm SD or proportions. BMI=body mass index; GPT=glutamate-pyruvate transaminase; GOT=glutamine oxaloacetic transaminase; $\gamma$-GT= $\gamma$-glutamyl transpeptidase; AKP=alkaline phosphatase; and $\mathrm{T}=$ body temperature. Sex distribution was compared using Chi-square between groups. After a test for homogeneity of variances, inter-group comparisons were performed using one-way analysis of variance (ANOVA) followed by Student-Newman-Keuls tests for post-hoc multiple comparisons (homoscedaticity) or using nonparametric Kruskal-Wallis tests followed by Conover's $\mathrm{t}$ tests for multiple comparisons (heteroscedaticity). \# $\mathrm{P}<0.05$, group I or group II versus III. $* \mathrm{P}<0.05$, group I versus group II. 
There were two major findings in the present study. The most notable finding is, compared with patients without hepatic disease, patients with cholestasis experienced prolonged duration time and longer recovery index after repeated doses of rocuronium, despite normal onset time after the initial dose. Second, cholestatic patients with or without hepatocellular injury represented the similar pharmacodynamics of rocuronium.

Heier and colleagues (11) and Caldwell and colleagues (12) concluded that hypothermia can increase the duration time and the recovery index of vecuronium, but the body temperature at the end of the trial in the three groups had no significant difference in the present study. Jellish and colleagues (14) found that spontaneous recovery of rocuronium is prolonged after the infusion of rocuronium more than $5 \mathrm{~h}$ in neurosurgical procedures. The time frame for the present study protocol was only $140 \mathrm{~min}$ or so and the duration time didn't prolong in patients without hepatic disease after repeated doses, however, the duration time was significantly prolonged in cholestatic patients after repeated doses. The more times rocuronium was repeatedly administered, the longer duration time the cholestatic patients experienced.

The elimination of rocuronium is via the liver and the kidney. Khuenl-Brady and colleagues (4) designed an animal study and concluded that rocuronium is mainly eliminated via biliary excretion with a small fraction $(10 \%)$ of rocuronium in urine. Proost and colleagues (5) also observed that the urinary excretion of rocuronium and metabolites is less than $10 \%$ and most of rocuronium is directly excreted into bile with very small amounts of metabolites. Sandker and colleagues (6) demonstrated that the isolated human hepatocytes in culture seemed to uptake and excrete rocuronium swiftly. Pharmacokinetic and pharmacodynamic studies in humans suggested that the action duration of rocuronium may be prolonged to a greater extent in patients with hepatic disease than in patients with renal disease (3). All the patients involved in the present study had no significant renal dysfunction; thereafter the pharmacodynamics difference among the groups should be related to the different liver status.

Rocuronium is taken up into the liver by a carrier-mediated active transport system $(15,16)$. There were some studies regarding pharmacokinetics and pharmacodynamics of rocuronium in cirrhotic patients (9-11), and these studies concluded that the increased onset time of rocuronium was significantly prolonged because of the increased central volume of distribution of rocuronium (9-11). Servin and colleagues (9) found that cirrhotic patients present a prolonged duration time after receiving five repeated doses because of a lower clearance of rocuronium. Van Miert and colleagues (10) and Khalil and colleagues (11) also demonstrated similar results and implied that the liver status is somewhat important for the clearance of rocuronium.

For these reasons, we presumed that the same onset and duration time of the initial dose was due to the distribution and the elimination of rocuronium among plasma, liver parenchymal, and bile, and the bile concentrations of rocuronium not being saturated after the initial dose was administered. However, after repeated doses, the bile concentrations are nearly saturated and the bile can't be easily excreted, the elimination of rocuronium is affected and the duration time and the recovery index are prolonged. In addition, there were somewhat differences without statistical significance in patients with or without hepatocellular injury. The key point of rocuronium elimination is the biliary excretion rather than the hepatocellular function, moreover, the liver parenchymal possesses an enormous compensatory potential (17). Driessen and colleagues (18) observed that 
Table 2. Onset time, duration time, and recovery index of rocuronium in cholestatic patients with hepatocellular injury (I), cholestatic patients without hepatocellular injury (II), and control group (without hepatic disease) (III). All received rocuronium $0.6 \mathrm{mg} / \mathrm{kg}$ initial dose followed by intermittent repeated administration of $0.15 \mathrm{mg} / \mathrm{kg}$.

\begin{tabular}{lccc}
\hline & Group I $(\mathrm{n}=20)$ & Group II $(\mathrm{n}=20)$ & Group III $(\mathrm{n}=20)$ \\
\hline Onset time (s) & $71 \pm 13$ & $70 \pm 12$ & $68 \pm 13$ \\
$1^{\text {st }}$ T25 (min) & $32 \pm 6$ & $31 \pm 6$ & $31 \pm 7$ \\
$2^{\text {nd }}$ T25 (min) & $22 \pm 4$ & $21 \pm 4$ & $22 \pm 4$ \\
$3^{\text {rd }}$ T25 (min) & $24 \pm 6$ & $23 \pm 6$ & $22 \pm 4$ \\
$4^{\text {th }}$ T25 (min) & $26 \pm 6$ & $25 \pm 5$ & $22 \pm 5$ \\
$5^{\text {th }}$ T25 (min) & $31 \pm 8 \# *$ & $28 \pm 5 \# *$ & $21 \pm 5$ \\
$6^{\text {th }}$ T25 (min) & $38 \pm 11 \# *$ & $33 \pm 9 \# *$ & $23 \pm 5$ \\
Recovery index (min) & $48 \pm 13 \#$ & $46 \pm 9 \#$ & $24 \pm 5$ \\
\hline
\end{tabular}

Data were expressed as mean \pm SD. $1^{\text {st }}, 2^{\text {nd }}, 3^{\text {rd }}, 4^{\text {th }}, 5^{\text {th }}$, and $6^{\text {th }}$ T25 represented the time from rocuronium $1^{\text {st }}, 2^{\text {nd }}, 3^{\text {rd }}$, $4^{\text {th }}, 5^{\text {th }}$, and $6^{\text {th }}$ administration to the recovery of the first twitch of the TOF response to $25 \%$, respectively. After a test for homogeneity of variances, inter-group and intra-group comparisons were performed using one-way ANOVA followed by Student-Newman-Keuls tests for post-hoc multiple comparisons. There was no significant difference between group I and group II. \# $\mathrm{P}<0.05$, group I or group II versus III (inter-group comparisons). $* \mathrm{P}<0.05,3^{\text {rd }}, 4^{\text {th }}$, $5^{\text {th }}$, or $6^{\text {th }} \mathrm{T} 25$ versus $2^{\text {nd }} \mathrm{T} 25$ (intra-group comparisons).

an end-stage renal failure patient showed accelerated recovery with enhanced elimination and clearance of rocuronium and suggested that increased hepatic binding of rocuronium rather than metabolism as the possible cause of this effect. Magorian and colleagues (19) also reported that hepatic impairment did not alter the plasma clearance of rocuronium.

There were some limitations in the present study. To interpret our data more reasonably, the plasma rocuronium concentrations should have been measured. This is important since there is no report on the pharmacokinetics of rocuronium in cholestatic patients. Hence, our explanation of the mechanism involved is speculative.

In conclusion, cholestatic patients experience prolonged duration time and longer recovery index after repeated use of rocuronium, despite normal onset time after the initial dose. Accordingly, attention to rocuronium effect is recommended and reduced maintenance doses are probably warranted in patients with cholestatic liver disease.

\section{REFERENCES}

[1]. Moore EW and Hunter JM. The new neuromuscular blocking agents: do they offer any advantages? Br J Anaesth, 87: 912-925, 2001.

[2]. Sparr HJ, Beaufort TM, and Fuchs-Buder T. Newer neuromuscular blocking agents: how do they compare with established agents? Drugs, 61: 919-942, 2001.

[3]. Bevan DR. Rocuronium bromide and organ function. Eur J Anaesthesiol Suppl, 9: 87-91, 1994.

[4]. Khuenl-Brady K, Castagnoli KP, Canfell PC, Caldwell JE, Agoston S, and Miller RD. The neuromuscular blocking effects and pharmacokinetics of ORG 9426 and ORG 9616 in the cat. Anesthesiology, 72: 669-674, 1990.

[5]. Proost JH, Wierda JM, Houwertjes MC, 
Roggeveld J, and Meijer DK. Structure-pharmacokinetics relationship of series of aminosteroidal neuromuscular blocking agents in the cat. J Pharmacol Exp Ther, 292: 861-869, 2000.

[6]. Sandker GW, Weert B, Olinga P, Wolters H, Slooff MJ, Meijer DK, and Groothuis GM. Characterization of transport in isolated human hepatocytes. A study with the bile acid taurocholic acid, the uncharged ouabain and the organic cations vecuronium and rocuronium. Biochem Pharmacol, 47: 2193-2200, 1994.

[7]. Hungness ES and Soper NJ. Management of common bile duct stones. J Gastrointest Surg, 10: 612-619, 2006.

[8]. Rosseland AR and Glomsaker TB. Asymptomatic common bile duct stones. Eur J Gastroenterol Hepatol, 12: 1171-1173, 2000.

[9]. Servin FS, Lavaut E, Kleef U, and Desmonts JM. Repeated Doses of Rocuronium Bromide Administered to Cirrhotic and Control Patients Receiving Isoflurane: A Clinical and Pharmacokinetic Study. Anesthesiology, 84: 1092-1100, 1996.

[10]. van Miert MM, Eastwood NB, Boyd AH, Parker CJ, and Hunter JM. The pharmacokinetics and pharmacodynamics of rocuronium in patients with hepatic cirrhosis. Br J Clin Pharmacol, 44: 139-144, 1997.

[11]. Khalil M, D'Honneur G, Duvaldestin P, Slavov V, De Hys C, and Gomeni R. Pharmacokinetics and pharmacodynamics of rocuronium in patients with cirrhosis. Anesthesiology, 80: 1241-1247, 1994.

[12]. Heier T, Caldwell JE, Sessler DI, and Miller RD. Mild intraoperative hypothermia increases duration of action and spontaneous recovery of vecuronium blockade during nitrous oxide-isoflurane anesthesia in humans. Anesthesiology, 74: 815-819, 1991.

[13]. Caldwell JE, Heier T, Wright PM, Lin S,
McCarthy G, Szenohradszky J, et al. Temperature-dependent pharmacokinetics and pharmacodynamics of vecuronium. Anesthesiology, 92: 84-93, 2000.

[14]. Jellish WS, Brody M, Sawicki K, and Slogoff S. Recovery from neuromuscular blockade after either bolus and prolonged infusions of cisatracurium or rocuronium using either isoflurane or propofol-based anesthetics. Anesth Analg, 91: 1250-1255, 2000.

[15]. Schwarz LR and Watkins JB 3rd. Uptake of taurocholate, a vecuronium-like organic cation, ORG 9426, and ouabain into carcinogen-induced diploid and polyploid hepatocytes obtained by centrifugal elutriation. Biochem Pharmacol, 43: 1195-1201, 1992.

[16]. Smit JW, Duin E, Steen H, Oosting R, Roggeveld J, and Meijer DK. Interactions between P-glycoprotein substrates and other cationic drugs at the hepatic excretory level. $\mathrm{Br}$ J Pharmacol, 123: 361-370, 1998.

[17]. Braun KM and Sandgren EP. Liver disease and compensatory growth: unexpected lessons from genetically altered mice. Int J Dev Biol, 42: 935-942, 1998.

[18]. Driessen JJ, Robertson EN, Booij LH, and Vree TB. Accelerated recovery and disposition from rocuronium in an end-stage renal failure patient on chronic anticonvulsant therapy with sodium valproate and primidone. $\mathrm{Br} \mathrm{J}$ Anaesth, 80: 386-388, 1998.

[19]. Magorian T, Wood P, Caldwell J, Fisher D, Segredo V, Szenohradszky J, et al. The pharmacokinetics and neuromuscular effects of rocuronium bromide in patients with liver disease. Anesth Analg, 80: 754-759, 1995. 